\title{
An Unbalanced Data Classification Model Using Hybrid Sampling Technique for Fraud Detection
}

\author{
T. Maruthi Padmaja ${ }^{1}$, Narendra Dhulipalla ${ }^{1}$, P. Radha Krishna ${ }^{1}$, \\ Raju S. Bapi ${ }^{2}$, and A. Laha ${ }^{1}$ \\ ${ }^{1}$ Institute for Development and Research in Banking Technology (IDRBT), \\ Hyderabad, India \\ \{tmpadmaja, dnarendra, prkrishna, alaha\}@idrbt.ac.in \\ ${ }^{2}$ Dept of Computer and Information Sciences, \\ University of Hyderabad, India - 500046 \\ bapics@uohyd.ernet.in
}

\begin{abstract}
Detecting fraud is a challenging task as fraud coexists with the latest in technology. The problem to detect the fraud is that the dataset is unbalanced where non-fraudulent class heavily dominates the fraudulent class. In this work, we considered the fraud detection problem as unbalanced data classification problem and proposed a model based on hybrid sampling technique, which is a combination of random under-sampling and over-sampling using SMOTE. Here, SMOTE is used to widen the data region corresponding to minority samples and random under-sampling of majority class is used for balancing the class distribution. The value difference metric (VDM) is used as distance measure while doing SMOTE. We conducted the experiments with classifiers namely k-NN, Radial Basis Function networks, C4.5 and Naive Bayes with varied levels of SMOTE on insurance fraud dataset. For evaluating the learned classifiers, we have chosen fraud catching rate, non-fraud catching rate in addition to overall accuracy of the classifier as performance measures. Results indicate that our approach produces high predictions against fraud and non-fraud classes.
\end{abstract}

Keywords: Fraud detection, SMOTE, VDM, Hybrid Sampling and Data Mining.

\section{Introduction}

Fraud is defined as 'criminal deception, the use of false representations to gain an unjust advantage'. It is a costly problem for many industries such as banking and insurance and co-exist with the latest in technology. Fraud detection is a continuously evolving discipline and requires a tool that is intelligent enough to adapt to criminals' strategies and ever changing tactics to commit fraud.

We can reduce some of these losses by using data mining techniques and collecting the customer data from the organizations. In general, fraud detection is 
a binary classification problem. However, in reality it is an $n$-class problem as fraud can be done in various ways and each modus operandi is different from the other. Compared to non-fraud data distribution, the fraud data is sparsely distributed. So it is extremely difficult to extract the fraud patterns in this scenario. In this work, we consider fraud detection as an unbalanced data classification problem where the majority samples (non-fraud samples) outnumber the minority samples (fraud samples). Usually, the classification algorithms exhibit poor performance while dealing with unbalanced datasets and results are biased towards the majority class. Hence, an appropriate model is needed to classify unbalanced data, especially for the fraud detection problem. For these type of problems, we cannot rely upon the accuracy of the classifier because the cost associated with fraud sample being predicted as a non-fraud sample is very high. The performance measures that can be used here are cost based metrics and ROC analysis.

In this paper, we proposed a model for fraud detection that uses hybrid sampling technique, which is a combination of random under-sampling and Synthetic Minority Over-sampling Technique (SMOTE [2]) to oversample the data. Here we have used Value Difference Metric (VDM [10]) as a distance measure in SMOTE. We identified optimal classifier based on its True Positive (TP) rate, True Negative $(T N)$ rate and consider accuracy as one of the measures.

The remainder of this paper is organized as follows. In Section 2 we present the brief literature review of the problem. Section 3 describes the proposed model and experimental setup. Section 4 provides the results and discussion. In section 5 we conclude the paper.

\section{Related Work}

In this section, we describe the related work from two perspectives: (i) The techniques available to handle the unbalanced data, and (ii) Available methods for fraud detection.

Some of the techniques available to handle the unbalanced data are: (a) oneclass classification, (b) sampling techniques (c) ensembling of classifiers and (d) rule based approaches. Foster [7] presented a review on the issues related to unbalanced data classification. From the experiments on 25 unbalanced data sets at different unbalanced levels (20 of them are from UCI), Weiss and provost [14] concluded that the natural distribution is not usually the best distribution for learning. Kubat and Matwin [9] did selective under-sampling of majority class by keeping minority classes fixed. They categorized the minority samples into some noise overlapping, the positive class decision region, borderline samples, redundant samples and safe samples. By using Tomek links concept, which is a type of data cleaning procedure used for under-sampling, they deleted the borderline majority samples. Kubat [8] proposed SHRINK system which searches for "best positive regions" among the overlapping regions of the majority and minority classes. Domingos [5] compared 'Metacost', a general method for making classifiers cost sensitive, with under-sampling and over-sampling techniques. He 
identified 'Metacost' outperform both the sampling techniques and he also observed that majority under-sampling is preferable than minority over-sampling technique. Chawla et al [2] proposed Synthetic Minority Over-sampling technique (SMOTE). It is an over-sampling approach in which the minority sample is over-sampled by creating synthetic (or artificial) samples rather than by oversampling with replacement. The minority class is over-sampled by taking each minority class sample and introducing synthetic samples along the line segments joining any/all of the $k$ minority class' nearest neighbors. Depending upon the amount of over-sampling required, neighbors from the $k$ nearest neighbors are randomly chosen. This approach effectively forces the decision region of the minority class to become more general. Study of "whether over-sampling is more effective than under-sampling" and "which over-sampling or under-sampling rate should be used" was done by Estabrooks et al [6], who concluded that combining different expressions of the resampling approach is an effective solution.

An excellent survey of work on fraud detection has recently been done by Clifton [4]. This work defines the professional fraudster, formalizes the main types and subtypes of known fraud and presents the nature of data evidence collected within affected industries. Stolfo et al [11] outlined a metaclassifier system for detecting credit card fraud by merging the results obtained from the local fraud detection tools at different corporate sites to yield a more accurate global tool. Similar kind of work has been carried out by Stolfo et al [12] and elaborated by Chan et al [1] and Stolfo et al [13]. Their work described a more realistic cost model to accompany the different classification outcomes. Wheeler and Aitken [15] have also explored the combination of multiple classification rules. Clifton et al [3] proposed a fraud detection method, which uses stackingbagging approach to improve cost savings.

\section{Proposed Model}

Figure 1 depicts our proposed hybrid sampling model for fraud detection. The model is a combination of random under-sampling and over-sampling. It mainly works based on determining how much percentage of minority samples (original minority + artificial minority samples) and majority samples to add to the training set such that a classifier can achieve best $T P$ and $T N$ rates. Here, $T P$ is the number of fraud samples correctly classified and $T N$ is the number of non-fraud samples correctly classified. The uniqueness of our model lies in generating synthetic minority samples using SMOTE, doing different levels of SMOTE, varying original fraud samples to be added to the training set and validating the classifier models on entire dataset. The majority data is randomly under-sampled and the minority data is over-sampled using SMOTE to emphasize the minority data regions, which uses distance metric called VDM.

Here, our idea is to do guided search for identifying the optimal SMOTE factor in the data space. This optimal SMOTE factor is dataset dependent. First we select some initial SMOTE factor for the data space and apply this SMOTE factor on varying minority original fraud percentages, continue this search by 


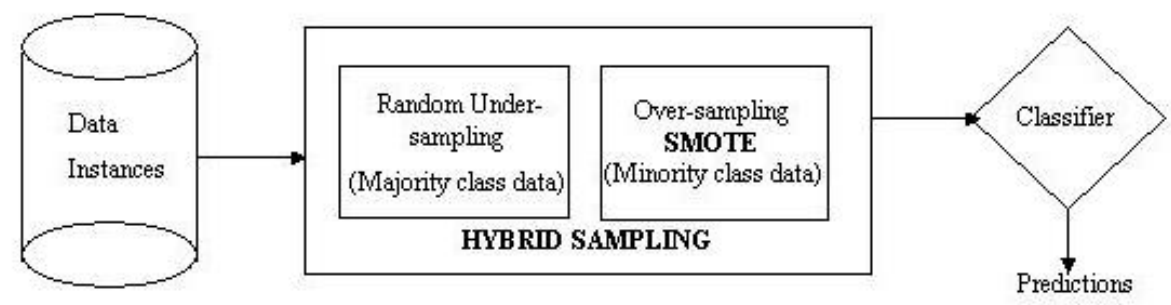

Fig. 1. Hybrid sampling model for fraud detection

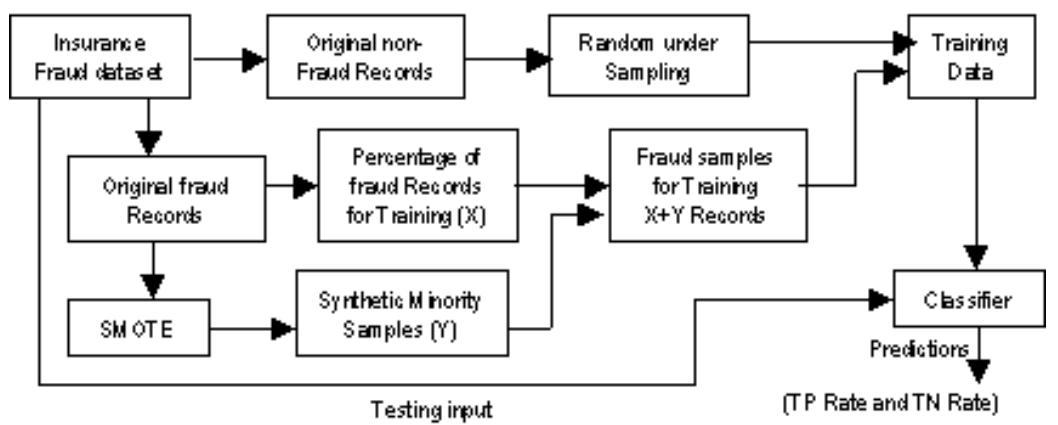

Fig. 2. Generation of samples for classification

systematically increasing SMOTE factor from initial value to the optimal SMOTE factor. At the optimal SMOTE point the underlying classifier exhibits best TP and $T N$ rates. As the dataset we are experimenting contains nominal attributes, we used VDM distance measure, suitable for nominal attributes in finding the nearest neighbors while doing SMOTE. Using this distance measure two values are considered to be closer if they have more similar classifications (i.e., more similar correlations with the output classes), regardless of what order the values may be given in.

We varied the number of synthetic minority samples (generated using SMOTE) to be given to the classifier by doing different levels of SMOTE and compared the effect of this on various classifiers. This is to know, how classifiers behave with respect to increase in number of minority samples added to the training set, i.e., by widening the boundary across the class region. In the presence of unbalanced datasets with unequal error costs, it is appropriate to use TP rate and $T N$ rate than accuracy alone.

Figure 2 shows the process of generating training samples for training the classifier. Initially, the fraud samples and non-fraud samples are separated into two different data sets and applied SMOTE on fraud samples for the given level of SMOTE factor. For example, if we specify SMOTE factor as 5 and input fraud samples are $\mathrm{x}$, then artificial fraud samples generated after SMOTE are $5 x$. Generally the choice of optimal SMOTE factor is data dependent. For the 
dataset under consideration, the class distribution of non-fraud (or majority samples) and fraud (or minority samples) samples is 94:6. So for experiments we considered SMOTE factors of 5,7,9,11 and 15. The training dataset is an amalgamation of artificially generated fraud samples, original fraud samples and non-fraud samples. We varied the Original Fraud Data Percentage (OFD percentage or rate), i.e., number of original fraud samples to be added to training, ranging from 0 to 75 . Similarly non-fraud samples were randomly under sampled from non-fraud data set in such a way that class distribution for training becomes 50:50. In this work, we have chosen classifiers namely k-NN, Radial Basis Function networks, C4.5 and Naive Bayes. Total experiments conducted are 100; 25 for each classifier by doing different levels of SMOTE and varying OFD rate.

\section{Experimental Results and Discussion}

Experiments are conducted on an insurance dataset used in [3]. The data set pertains to automobile insurance and it contains 15421 samples, out of which 11338 samples are from January-1994 to December-1995 and remaining 4083 samples are form January-1996 to December -1996. There are 30 independent attributes and one dependent attribute (class label). Here, six are numerical attributes and remaining are categorical attributes. The class distribution of non-fraud and fraud is 94:6 which indicates that the data is highly unbalanced. We discarded the attribute PolicyType, because it is an amalgamation of existing attributes VehicleCategory and BasePolicy. Further, we created three attributes namely weeks-past, is-holidayweek-claim and age-price-wsum to improve the predictive accuracy of classifiers as suggested in [3]. So the total numbers of attributes used are 33. All the numerical attributes are discrete in nature and thus converted into categorical attributes in order to compute the distance between the samples using VDM.

We implemented SMOTE in MATLAB 7.0 and used the WEKA3.4 toolkit for experimenting with four classification algorithms selected. We computed classification accuracy, $T P$ rate and $T N$ rate by varying OFD rate on each SMOTE factor. We have set $k$,i.e., the number of nearest neighbors to be selected while doing SMOTE, as 7,9,11,13 and 17 for SMOTE factors 5,7,9,11 and 15 respectively. Tables $1,2,3$ and 4 show the $T P$ rate and $T N$ rate for $\mathrm{k}-\mathrm{NN}, \mathrm{RBF}$ neural network, C4.5 and Naive Bayes classifiers respectively. It was observed from the experiments that the accuracy is more than $90 \%$ in more than 40 cases out of 100 experiments. Figures 3 to 6 shows how classifiers behave when we change OFD rate and SMOTE factor. The $\mathrm{X}$-axis represents the OFD rate and $\mathrm{Y}$-axis represents the classification accuracy.

The observations from experiments conducted are as follows. K-NN has recorded slight decrease in accuracy with increase in OFD rate for fixed SMOTE factor. Accuracy of the classifier is improved when SMOTE factor is increased, but with increase in the OFD rate, it has decreased slightly. This is because non-fraud catching rate has decreased with increase in OFD rate and non-fraud samples account for more percentage in the test set. It has shown a best fraud 


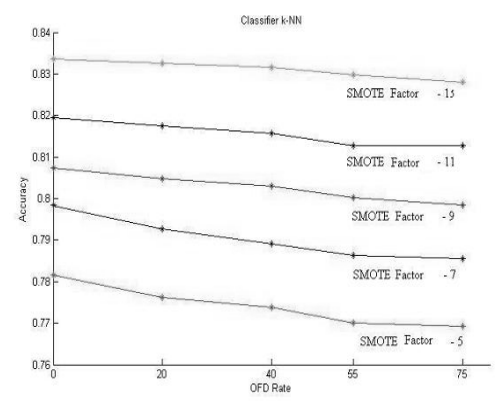

Fig. 3. Plot of OFD rate Vs Accuracy for classifier $\mathrm{k}-\mathrm{NN}(\mathrm{k}=10)$

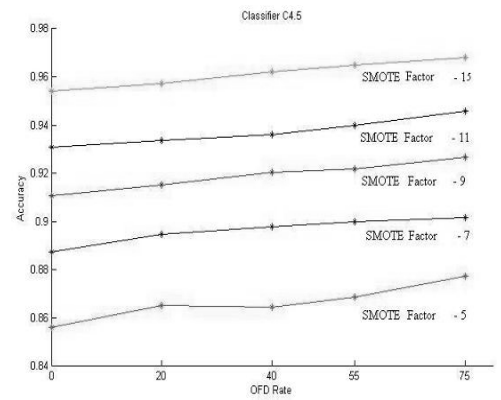

Fig. 5. Plot of OFD rate VS Accuracy for classifier $\mathrm{C} 4.5$

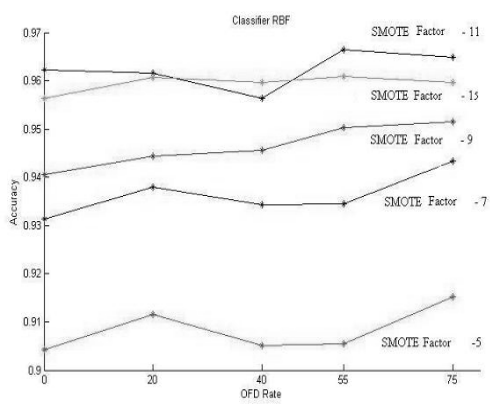

Fig. 4. Plot of OFD rate Vs Accuracy for classifier $\mathrm{RBF}$

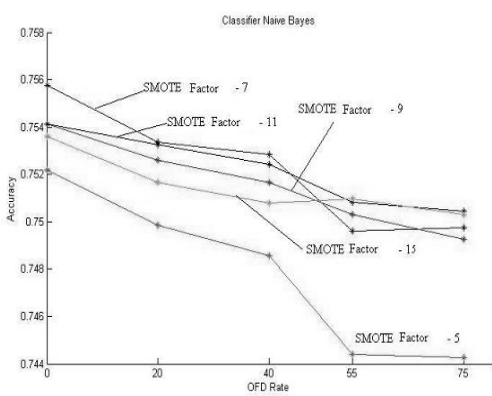

Fig. 6. Plot of OFD rate Vs Accuracy for classifier Naive Bayes

catching rate of $99.9 \%$. In all the 25 experiments conducted on this classifier, the fraud catching rate of more than $95 \%$ (Table 1 ). This may be due to inherent use of k-NN in generating artificial minority samples by SMOTE. RBF has got accuracy and non-fraud catching rate of more than $90 \%$ in all the 25 cases. Fraud catching rate is also equally good which is between $86.2 \%$ and $96.6 \%$ (Table 2). With respect to increase in OFD rate, $\mathrm{C} 4.5$ has recorded a slight increase in accuracy for all SMOTE Levels. It has shown good improvement in accuracy from 0.856 to 0.967 by increasing SMOTE factor ranging from 5 to 15 . In the case of Naive Bayes classifier, accuracy has reduced slightly by increase in OFD rate for each SMOTE factor. This classifier has not shown any improvement in accuracy for varied SMOTE factor and OFD rate (Table 4). For all classifiers at all SMOTE factors, with increase in OFD rate, $T P$ rate has increased slightly but there is not much impact on $T N$ rate.

We also ranked the classifiers according to accuracy, fraud catching rate and non-fraud catching rate (results are not shown due to paucity of space). Classifiers $\mathrm{C} 4.5$ and $\mathrm{RBF}$ have performed very well compared to the remaining two classifiers. Though, Naive Bayes classifier works well in general, in our experiments it has shown poor performance on the data set under consideration com- 
Table 1. $T P$ and $T N$ values for $\mathrm{k}-\mathrm{NN}$

\begin{tabular}{|l|c|c|c|c|c|c|}
\hline SMOTE & & \multicolumn{5}{|c|}{ OFD rate } \\
\cline { 3 - 7 } factor & & 0 & 20 & 40 & 55 & 75 \\
\hline 5 & $T P$ & .94 & .95 & .95 & .95 & .96 \\
& $T N$ & .77 & .76 & .76 & .75 & .75 \\
\hline 7 & $T P$ & .97 & .97 & .97 & .97 & .98 \\
& $T N$ & .78 & .78 & .77 & .77 & .77 \\
\hline 9 & $T P$ & .98 & .99 & .99 & .99 & .99 \\
& $T N$ & .79 & .79 & .79 & .78 & .78 \\
\hline \multirow{2}{*}{11} & $T P$ & .99 & .99 & .99 & .99 & .99 \\
& $T N$ & .80 & .80 & .80 & .80 & .80 \\
\hline \multirow{2}{*}{15} & $T P$ & .99 & .99 & .99 & .99 & .99 \\
& $T N$ & .82 & .82 & .82 & .81 & .81 \\
\hline \hline
\end{tabular}

Table 3. $T P$ and $T N$ values for $\mathrm{C} 4.5$

\begin{tabular}{|l|c|c|c|c|c|c|}
\hline \multirow{2}{*}{$\begin{array}{l}\text { SMOTE } \\
\text { factor }\end{array}$} & & \multicolumn{5}{|c|}{ OFD rate } \\
\cline { 3 - 7 } 5 & $T P$ & .67 & 20 & 40 & 55 & 75 \\
\hline 5 & $T N$ & .86 & .87 & .87 & .83 & .86 \\
& $T P$ & .70 & .76 & .80 & .83 & .87 \\
\hline \multirow{2}{*}{7} & $T N$ & .89 & .90 & .90 & .90 & .90 \\
\hline 9 & $T P$ & .71 & .77 & 81 & .83 & .87 \\
& $T N$ & .92 & .92 & .92 & .92 & .93 \\
\hline \multirow{2}{*}{11} & $T P$ & .72 & .77 & .80 & .84 & .89 \\
& $T N$ & .94 & .94 & .94 & .94 & .94 \\
\hline \multirow{2}{*}{15} & $T P$ & .73 & .78 & .82 & .85 & .88 \\
& $T N$ & .96 & .96 & .97 & .97 & .97 \\
\hline \hline
\end{tabular}

Table 2. $T P$ and $T N$ values for $\mathrm{RBF}$

\begin{tabular}{|l|l|c|c|c|c|c|}
\hline \multirow{2}{*}{$\begin{array}{l}\text { SMOTE } \\
\text { factor }\end{array}$} & & \multicolumn{5}{|c|}{ OFD rate } \\
\cline { 3 - 7 } 5 & & 0 & 20 & 40 & 55 & 75 \\
\hline \multirow{2}{*}{7} & $T P$ & .86 & .88 & .90 & .92 & .96 \\
& $T N$ & .90 & .91 & .90 & .90 & .91 \\
\hline \multirow{2}{*}{9} & $T P$ & .88 & .90 & .94 & .94 & .96 \\
& $T N$ & .93 & .94 & .93 & .93 & .94 \\
\hline \multirow{2}{*}{11} & $T P$ & .88 & .90 & .92 & .93 & .96 \\
& $T N$ & .94 & .94 & .94 & .95 & .95 \\
\hline \multirow{2}{*}{15} & $T P$ & .91 & .94 & .93 & .95 & .96 \\
& $T N$ & .96 & .96 & .95 & .96 & .96 \\
\hline \hline
\end{tabular}

Table 4. $T P$ and $T N$ values for Naive Bayes

\begin{tabular}{|l|l|l|l|l|l|l|}
\hline SMOTE & & \multicolumn{5}{|c|}{ OFD rate } \\
\cline { 3 - 7 } factor & & 0 & 20 & 40 & 55 & 75 \\
\hline 5 & $T P$ & .59 & .60 & .61 & .62 & .65 \\
& $T N$ & .76 & .75 & .75 & .75 & .75 \\
\hline 7 & $T P$ & .59 & .60 & .60 & .61 & .63 \\
& $T N$ & .76 & .76 & .76 & .75 & .75 \\
\hline 9 & $T P$ & .59 & .60 & .60 & .61 & .62 \\
& $T N$ & .76 & .76 & .76 & .75 & .75 \\
\hline \multirow{2}{*}{11} & $T P$ & .59 & .60 & .60 & .60 & .61 \\
& $T N$ & .76 & .76 & .76 & .76 & .75 \\
\hline \multirow{2}{*}{15} & $T P$ & .60 & .60 & .60 & .60 & .61 \\
& $T N$ & .76 & .76 & .76 & .76 & .75 \\
\hline \hline
\end{tabular}

pared to other classifiers. Generally, if we consider only accuracy as performance measure for fraud detection, classifiers applied on original dataset will give more accuracy by predicting all fraud samples as non-fraud samples. But high classification accuracy is achieved using proposed model by increasing fraud catching rate, besides maintaining high non-fraud catching rate.

\section{Conclusion}

In this paper, we explored the existing methods for fraud detection and proposed a new model based on hybrid sampling approach. Our model uses superior approach SMOTE to oversample the fraud data. We observed that by using SMOTE, the fraud regions emphasized well so that the classifiers can able to learn from the fraud class. Experiments are conducted on insurance data set for four classifier namely k-NN, RBF neural networks, C4.5 and Naive Bayes. Results demonstrated that our model is efficient for fraud detection . For instance, when we applied RBF by taking SMOTE factor as 11 and kept OFD rate as 55 , we got non-fraud catching rate of $96.7 \%$ ( $T N$ rate), fraud catching rate of 95.9\% (TP rate) and the accuracy of $96.6 \%$. Thus, intelligent use of SMOTE for generating artificial fraud samples resulted in improving the accuracy, non-fraud catching rate and fraud catching rate. Though the proposed model implemented for insurance domain, it can be applicable to other domains as well for fraud detection. 


\section{References}

1. Chan, P., Fan, W., Prodromidis, A., Stolfo, S.: Distributed Data Mining in Credit Card Fraud Detection. IEEE Intelligent Systems 14, 67-74 (1999)

2. Chawla, N.V., Bowyer, K.W., Hall, L.O., Kegelmeyer, W.P.: SMOTE: Synthetic Minority Over-sampling Technique. JAIR 16, 324-357 (2004)

3. Phua, C., Damminda, A., Lee, V.: Minority Report in Fraud Detection: Classification of Skewed Data. Sigkdd Explorations 6(1) (2004)

4. Clifton phua Lee, V., Smith, K., Gayler, R.: A Comprehensive Survey of Data Mining-based Fraud Detection Research. In: Artificial Intelligence review (2005)

5. Domingos, P.: Metacost: A General Method for Making Classifiers Cost-sensitive. In: Proceedings of the Fifth ACM SIGKDD International Conference on Knowledge Discovery and Data Mining, pp. 155-164. ACM Press, New York (1999)

6. Estabrooks, A., Jo, T., Japkowicz, N.: A Multiple Resampling Method for Learning from Imbalances Data Sets. Computational Intelligence 20(1) (2004)

7. Foster, P.: Machine learning from imbalanced data sets. Invited paper for the AAAI 2000 Workshop on Imbalanced Data Sets (2000)

8. Kubat, M., Holte, R., Matwin, S.: Machine Learning for the Detection of Oil Spills in Satellite Radar Images. Machine Learning 30, 195-215 (1998)

9. Kubat, M., Matwin, S.: Addressing the Curse of Imbalanced Training Sets: One Sided Selection. In: Proceedings of the Fourteenth International Conference on Machine Learning, Nashville, Tennesse, pp. 179-186. Morgan Kaufmann, San Francisco (1997)

10. Wilson, R., Tony,: Improved Heterogeneous Distance Functions. JAIR 6, 1-34 (1997)

11. Stolfo, J., Fan, D.W., Lee, W., Prodromidis, A.L.: Credit card fraud detection using meta-learning: Issues and initial results. In: AAAI Workshop on AI Approaches to Fraud Detection and Risk Management, pp. 83-90. AAAI Press, Menlo Park, CA (1997)

12. Stolfo, S., Andreas, L.P., Tselepis, S., Lee, W., Fan, D.W.: JAM: Java agents for meta-learning over distributed databases. In: AAAI Workshop on AI Approaches to Fraud Detection and Risk Management, pp. 91-98. AAAI Press, Menlo Park, CA (1997)

13. Wei Fan, S., Lee, W., Prodromidis, A., Chan, P.: Cost-based modeling for fraud and intrusion detection: Results from the JAM Project. In: Proceedings of the DARPA Information Survivability Conference and Exposition 2, pp. 130-144. IEEE Computer Press, New York (1999)

14. Weiss, G., Provost, F.: The Effect of Class Distribution on Classifier Learning. Technical Report ML-TR-43, Department of Computer Science, Rutgers University (January 2001)

15. Wheeler, R., Aitken, S.: Multiple algorithms for fraud detection. Knowledge-Based Systems 13(2/3), 93-99 (2000) 